\title{
Complementary Beamlines at the 4th Generation Sirius Synchrotron for Hierarchical Analyses of Geochemical Systems
}

DEAN HESTERBERG, HÉLIO TOLENTINO AND HARRY WESTFAHL JR.

Brazilian Synchrotron Light Laboratory (LNLS-CNPEM)

Presenting Author: dean.hesterberg@lnls.br

The new 4th generation Sirius synchrotron source at LNLS is designed with multiple beamlines that will provide scattering, imaging, and spectroscopy capabilities spanning length scales from centimeters to angstroms. This presentation will highlight some complementary beamline capabilities that will be particularly useful for studying the behavior of metals and nutrients in geochemical systems such as soils. MOGNO is a hard X-ray zoom tomography beamline with fields of view ranging from $85 \mathrm{~mm}$ to $150 \mu \mathrm{m}$ and corresponding spatial resolutions of $55 \mu \mathrm{m}$ to $120 \mathrm{~nm}$. CATERETÊ is a coherent scattering/coherent diffraction imaging (CDI) beamline for highresolution $(\sim 10 \mathrm{~nm})$ full field imaging of nano and microstructure within particles $<30 \mu \mathrm{m}$, or ptychography on larger samples. Two other beamlines being developed for highenergy (JATOBÁ) and small-angle (SAPUCAIA) X-ray scattering add angstrom level pair-distribution function (pdf) analysis and nanometer to micron-scale morphological analysis of particles in aqueous suspensions by SAXS. The PAINEIRA beamline provides powder X-ray diffraction in either highthroughput or high-resolution modes. CARNAÚBA is a sophisticated imaging and spectroscopy beamline covering an energy range from 2 to $15 \mathrm{keV}$, with target beam spot sizes from 150 to $30 \mathrm{~nm}$. Multiple detectors placed around a sample provide spatially resolved $2 \mathrm{D}$ or $3 \mathrm{D}$ diffraction and chemical imaging capabilities in either transmission (STXM) or fluorescence $(\mu / \mathrm{n}$ $\mathrm{XRF}$ ) modes, as well as ptychography. A high-stability monochromator adds speciation analysis by $\mu / \mathrm{n}$-X-ray absorption spectroscopy (XAS). The high intensity, highly coherent X-rays produced by Sirius generally allow rapid (seconds to minutes) analysis of typical samples on these beamlines. QUATI is a quick-XAS beamline, with an energy scanning rate of 50 to 500 $\mathrm{ms} / \mathrm{scan}$ for in-situ, time-resolved chemical speciation. Collectively, these beamlines provide both physical and chemical information on geochemical samples across a range of scales to define mechanisms of hierarchical processes driving environmental impacts of trace elements and nutrients. 